\title{
Erratum to: Siansivirga jejunensis sp. nov., isolated from seawater of Jeju Island in Korea and emendation of the genus Siansivirga
}

\author{
Ji-Min Park • Sang-Keun Han • Dong-Geol Lee • \\ Heecheol Kang • Martha E. Trujillo • \\ Dong-Kyu Lee
}

Published online: 17 December 2014

(C) Springer International Publishing Switzerland 2014

\section{Erratum to: Antonie van Leeuwenhoek (2014) 106:763-769 \\ DOI 10.1007/s10482-014-0246-9}

Subsequent to the publication of the above paper it has been brought to our attention that the species epithet proposed for the taxon represented by strain $\mathrm{JHH}-2^{\mathrm{T}}$ is not grammatically correct, as "jejunensis" should have been proposed as "jejuensis". We here propose the corrected name for the taxon represented by strain $\mathrm{JHH}-2^{\mathrm{T}}$. Moreover we provide the corrected strain

The online version of the original article can be found under doi:10.1007/s10482-014-0246-9.

J.-M. Park · D.-G. Lee ( $₫)$

Microbial Metabolite Division, GFC, 309 GFC, Tower-

Dong, Heungdeok IT Valley, 13, Heungdeok 1-Ro,

Giheung-gu, Yongin 446-908, Gyeonggi-do,

Republic of Korea

e-mail: nalpharam@naver.com

J.-M. Park

e-mail: rhaoddl@hanmail.net

S.-K. Han

Skin Research Institute, Korea Kolmar Corporation,

12-11, Dukgogae-gil, Sejong, Republic of Korea

e-mail:96183@Kolmar.co.kr

H. Kang

GFC R\&D Center, GFC, 309 GFC, Tower-Dong,

Heungdeok IT Valley, 13, Heungdeok 1-Ro, Giheung-gu,

Yongin 446-908, Gyeonggi-do, Republic of Korea

e-mail: cmkorea@unitel.co.kr code for the deposition of the strain in the Korean Culture Center of Microorganisms.

\section{Description of Siansivirga jejuensis sp. nov.}

Siansivirga jejuensis (jejuensis: je.ju.en'sis. N.L. fem.adj. jejuensis, of or belonging to Jeju, the island from which the type strain was isolated).

The description of the species is as given for "Siansivirga jejunensis" in Park et al. (2014) Antonie van Leeuwenhoek 106:763-769.

The type strain is JHH- $2^{\mathrm{T}}\left(=\mathrm{KCCM} 90230^{\mathrm{T}}=\mathrm{JCM}\right.$ $19228^{\mathrm{T}}$ ).

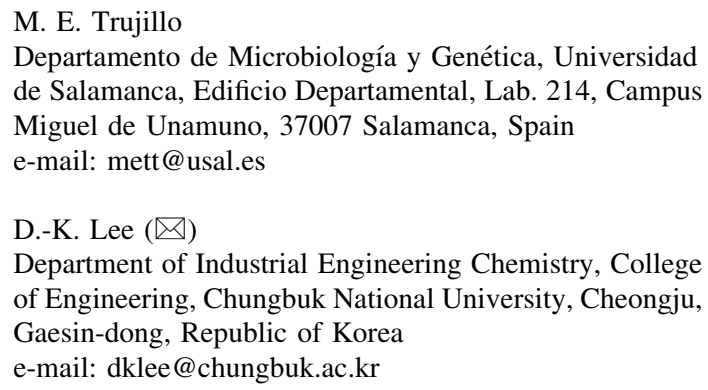

